\title{
Investigation of the association between miR-181b, Bcl-2 and LRIG1 in oral verrucous carcinoma
}

\author{
ZHI-YUAN DENG ${ }^{1}$, YUE-HONG WANG ${ }^{1}$, HONG-ZHI QUAN ${ }^{1}$, OU-SHENG LIU ${ }^{1}$, YI-PING LI ${ }^{2}$, \\ YUAN $\mathrm{LI}^{3}$, WU ZHU ${ }^{4}$, KRISHNA MUNNEE ${ }^{1,5}$ and ZHAN-GUI TANG ${ }^{1}$ \\ Departments of ${ }^{1}$ Oral and Maxillofacial Surgery and ${ }^{2}$ Prosthodontics, \\ Xiangya School and Hospital of Stomatology, Central South University, Changsha, Hunan 410078; \\ ${ }^{3}$ Department of Oral and Maxillofacial Surgery, Changsha Stomatological Hospital, Changsha, Hunan 410005; \\ ${ }^{4}$ Department of Oral and Maxillofacial Surgery, Yiyang Central Hospital, Yiyang, Hunan 413000, P.R. China; \\ ${ }^{5}$ Department of Plastic Surgery, Victoria Hospital, Quatre Bornes 230, Mauritius
}

Received July 8, 2015; Accepted July 21, 2016

DOI: $10.3892 / \mathrm{mmr} .2016 .5608$

\begin{abstract}
Abnormal expression of microRNAs (miRNAs) is involved in the development of and anti-apoptotic effects in various types of human cancer. However, miRNA-mediated regulation of oral verrucous carcinoma (OVC) remains to be elucidated. The present study aimed to investigate the expression of miR-181b in OVC and oral squamous cell carcinoma (OSCC). The expression levels of miR-181b were determined using reverse transcription-quantitative polymerase chain reaction. The expression levels of B-cell lymphoma 2 (Bcl-2) and leucine rich repeats and immunoglobulin like domains 1 (LRIG1), were evaluated using immunohistochemical staining. The correlation between Bcl-2 and LRIG1 expression was determined using a Pearson correlation analysis. The expression levels of miR-181b and $\mathrm{Bcl}-2$ in OVC were significantly higher compared with normal mucosal tissue (NM); however, lower compared with the OSCC. The key target of miR-181b was LRIG1 and it was significantly lower in OVC tissues compared with NM tissue; however this was higher when compared with OSCC tissue. The expression levels of Bcl-2 were correlated with expression levels of LRIG1 in OVC tissues. Therefore, LRIG1 may be associated with anti-apoptotic function in OVC tissues.
\end{abstract}

\section{Introduction}

Verrucous carcinoma was first reported in 1948 as a non-metastatic variant of squamous cell carcinoma and its unique biological behavior has attracted increasing research

Correspondence to: Professor Zhan-Gui Tang, Department of Oral and Maxillofacial Surgery, Xiangya School and Hospital of Stomatology, Central South University, 72 Xiangya Road, Changsha, Hunan 410078, P.R. China

E-mail: tangzhangui@aliyun.com

Key words: apoptosis, oral verrucous carcinoma, miRNA, Bcl-2, LRIG1 attention $(1,2)$. This tumor is frequently prevalent in the oral cavity; however, it may also occur in the throat, esophagus, nasal and paranasal sinuses, external genital organs and on the skin $(3,4)$. Oral verrucous carcinoma (OVC) proliferates slowly, with a high degree of differentiation and male smokers $>60$ years of age are the most commonly affected group (5). The present treatment options include surgery, chemotherapy, and radiotherapy or combination therapy. Although the disease has a good prognosis, the rate of recurrence is high (6). The primary etiologic factors for this tumor have been reported to be tobacco and betel nut chewing, and infection with the human papilloma virus (7). The genes encoding vascular endothelial growth factor, E-cadherin and murine double minute 2 are also important in the development of OVC (8-11); however, the molecular mechanism underlying their involvement in its pathogenesis remains to be elucidated.

A microRNA (miRNA) is an endogenous small non-coding RNA of 22 nucleotides in length, which negatively regulates target genes during translation (12). It is estimated that one-third of all miRNAs are involved in the regulation of cellular proliferation, apoptosis, DNA repair and various other physiological processes (13). Abnormal miRNA expression has been identified in various types of cancer, including breast, lung, liver, and head and neck cancer (14). Overexpression of miR-181b, a member of the miR-181s family, in tumor cells enhanced the growth and invasive capabilities of the tumor and was able to inhibit the apoptosis of these tumor cells. Thus, this has led to the identification of miR-181b as an oncogene in gastric cancer, liver cancer and oral squamous cell carcinoma (OSCC) (15-17). In addition, it has been reported that miR-181 is important for the malignant transformation of oral premalignant lesions $(17,18)$. It is of note, that there is evidence to the contrary, suggesting that miR-181 promotes the apoptosis of tumor cells via the downregulation of Bcl-2 expression levels, which has been has an anti-apoptotic function in various types of cancer $(19,20)$. The importance and expression level of miR-181b in OVC requires further investigation.

B-cell lymphoma 2 (Bcl-2) is an important regulator of apoptosis by blocking the release of cytochrome $c$ and inhibiting the caspases (21). Overexpression of Bcl-2 has been 
demonstrated in numerous types of cancer. The leucine-rich repeats and immunoglobulin-like domains 1 (LRIG1) protein may act as a master molecule, that regulates stem cells in various cancers and is able to inhibit the growth of different types of cancer. The mechanism underlying its inhibition of proliferation has been reported to involve the downregulation of Bcl-2 expression levels $(22,23)$. LRIG1 is also a target of miR-181b. Previous studies have determined that miR-181s is important for the apoptotic process of tumor cells (16). However, this claim is debatable and the exact molecular mechanism remains to be elucidated.

The present study investigated the expression of miR-181b and LRIG1 in OVC, OSCC para-tumor and normal mucosal tissues. It was determined that decreased expression levels of LRIG1 and increased expression levels of miR-181b were present in OVC. In addition, it was observed that expression levels of Bcl-2 were negatively correlated with the expression of LRIG1 in OVC.

\section{Materials and methods}

Tissue specimens. The study was approved by the ethics committee of Central South University (Changsha, China). A total of 30 samples were obtained from patients between June 2009 to January 2013, all underwent surgery in the Oral and Maxillofacial Surgery department at the Xiangya Hospital of Central South University (Changsha, China). The tumor samples and their corresponding adjacent tissues were obtained from 6 patients with exogenous OVC (a tumor the occurs on the surface of the oromaxillo-facial region) and 6 patients with well-differentiated OSCC. Normal tissues were obtained from 6 patients with maxillofacial trauma. The diagnosis was conducted by two independent pathologists. The patients did not receive preoperative medication and all were informed of the preoperative surgical planning and purpose of the experiment. Informed consent for the present study was obtained from each participant. In the OVC group, there were 4 males and 2 females with a mean age of $51 \pm 12.8$ years, in the OSCC group, there were 5 males and 1 female with mean age of $48 \pm 14.6$ years, in the NM group, there were 4 males and 2 females with mean age of $31 \pm 9.4$ years. All tissue samples were immediately frozen in liquid nitrogen and stored at $-80^{\circ} \mathrm{C}$ until used.

A total of 105 paraffin-embedded samples dating between 1996 and 2014 were obtained from archival specimens in the Department of Pathology, Xiangya Hospital, Central South University for immunohistochemistry (IHC), this included 15 OVC and 30 well-differentiated OSCC samples (including tumor samples and adjacent para-tumor tissues) and 15 normal oral mucosa samples. In the OVC group, there were 11 males and 4 females with a mean age of $54 \pm 11.3$ years, in the OSCC group, there were 26 males and 4 females with a mean age of $57 \pm 9.3$ years, in the NM group, there were 6 males and 9 females with a mean age of $35 \pm 13.3$ years.

Total RNA extraction and reverse transcription (RT). Total RNA was extracted from patient and control tissues using TRIzol reagent (Invitrogen; Thermo Fisher Scientific, Inc., Waltham, MA, USA) following the manufacturer's protocol. The concentration and quality of the RNA was determined using a Nanodrop 2000 spectrophotometer. First-strand cDNA

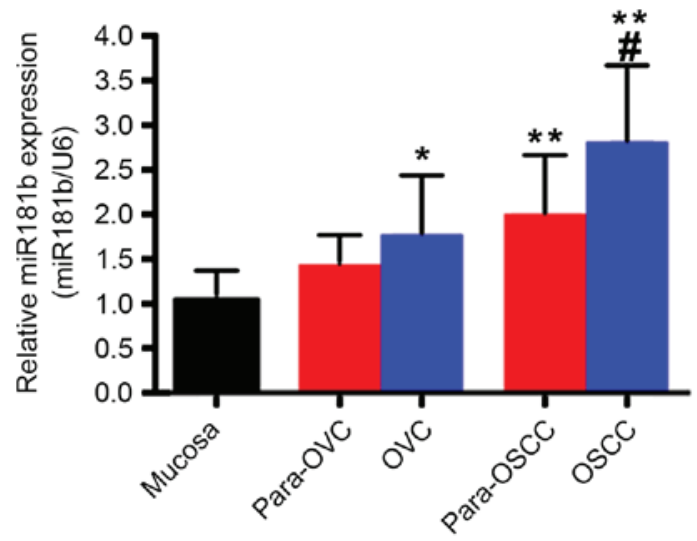

Figure 1. Expression of miR-181b in human NM, OVC and OSCC tissues. miR-181b expression in OVC and OSCC tissue samples was evaluated using reverse transcription-polymerase chain reaction and normalized using the expression level of U6. ${ }^{*} \mathrm{P}<0.05$, OVC vs. NM; ${ }^{* *} \mathrm{P}<0.01$, OSCC vs. NM, para-OSCC vs. NM; ${ }^{\#} \mathrm{P}<0.05$, OVC vs. OSCC. miR, microRNA; NM, normal oral mucosa; OVC, oral verrucous carcinoma; para-OVC, para-tumor tissues of oral verrucous carcinoma; OSCC, oral squamous cell carcinoma; Para-OSCC, para-tumor tissues of oral squamous cell carcinoma.

was synthesized using $2 \mu \mathrm{g}$ of total RNA and M-MLV reverse transcriptase (Promega Corporation, Madison, WI, USA). A DNase step was performed using DNase I (Invitrogen; Thermo Fisher Scientific, Inc.) Specific Bulge-Loop miRNA qPCR primers were obtained from RiboBio Co., Ltd. (Guangzhou, China).

Quantitative polymerase chain reaction $(q P C R)$. The expression levels of miR-181b and the internal reference gene U6 were determined using SYBR Premix Ex Taq II (Takara Biotechnology Co., Ltd., Dalian, China) on a TP800 thermocycler. The total reaction volume was $20 \mu \mathrm{l}$ and was composed of $10 \mu \mathrm{l}$ SYBR Master Mixture, $0.5 \mu \mathrm{l}$ forward primer, $0.5 \mu \mathrm{l}$ reverse primer, $1 \mu \mathrm{l}$ cDNA and $8 \mu \mathrm{ldd} \mathrm{d}_{2} \mathrm{O}$. The conditions for the reaction were: $95^{\circ} \mathrm{C}$ for $10 \mathrm{~min}, 95^{\circ} \mathrm{C}$ for $15 \mathrm{~s}, 55^{\circ} \mathrm{C}$ for $30 \mathrm{~s}, 70^{\circ} \mathrm{C}$ for $30 \mathrm{sec}, 40$ cycles from step 2 . The fluorescence signal was detected at $70^{\circ} \mathrm{C}$. The relative quantity of gene expression was calculated using the $2^{-\Delta \Delta \mathrm{Cq}}$ formula (24). The following primers were used for qPCR: miR-181b, forward 5'-CTTGGTACCGAG CTCTCCTAGAGCTCTGTTCGCCT-3', reverse 5'-TGCTGG ATATCTGCACGAACATTCACATGAGGGCG-3'; and U6, forward 5'- CTCGCTTCGGCAGCACA-3', reverse 5'-AAC GCTTCACGAATTTGCGT-3'.

IHC staining. Slices of $\sim 4 \mu \mathrm{m}$ were cut and dried at $60^{\circ} \mathrm{C}$ for $2.5 \mathrm{~h}$, then dewaxed and hydrated in accordance with routine procedures. The slices were boiled in $0.01 \mathrm{M}$ citric acid buffer solution (pH 6.0) for $90 \mathrm{sec}$ at high pressure. Droplets of 3\% hydrogen peroxide were placed on the slice and left standing at $37^{\circ} \mathrm{C}$ temperature for $20 \mathrm{~min}$ to remove endogenous peroxidase. The tissues were then blocked for non-specific binding with 10\% normal goat serum (OriGene Technologies, Inc., Beijing, China) for $1 \mathrm{~h}$ at $37^{\circ} \mathrm{C}$. Incubation with primary antibodies was performed overnight at $4^{\circ} \mathrm{C}$, the antibodies used were as follows: Monoclonal mouse anti-Bcl-2 (1:200, Cell Signaling Technology, Danvers, MA, USA; cat. no. 15071S), polyclonal rabbit anti-LRIG1 (1:150; Abcam, Cambridge, UK; 
A

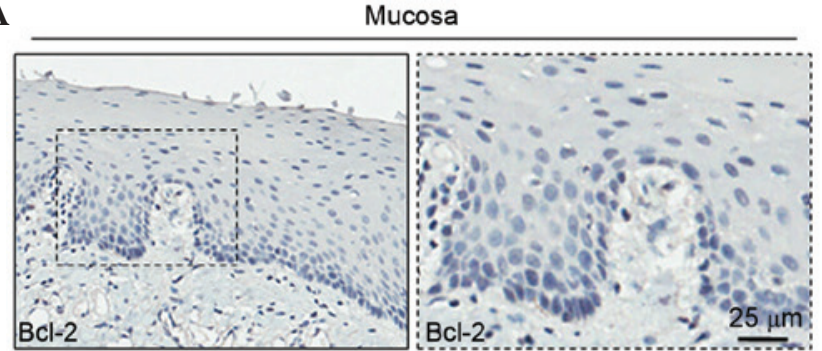

C

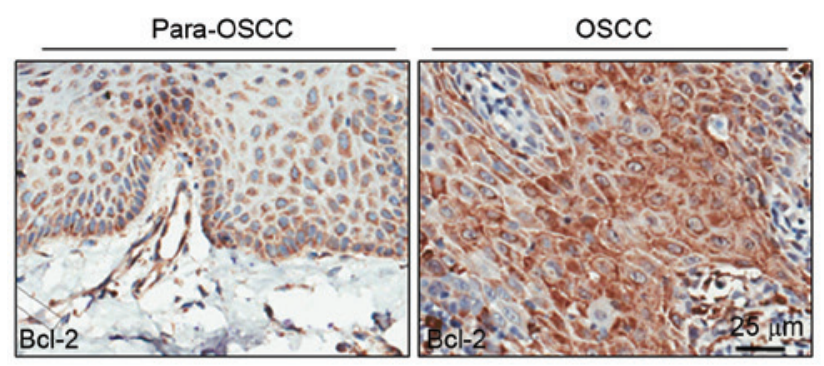

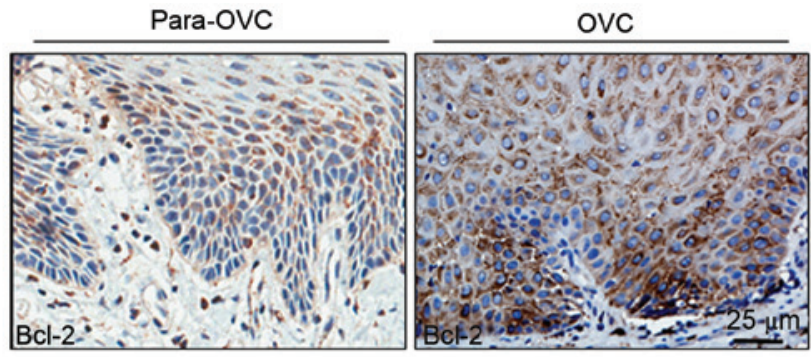

D

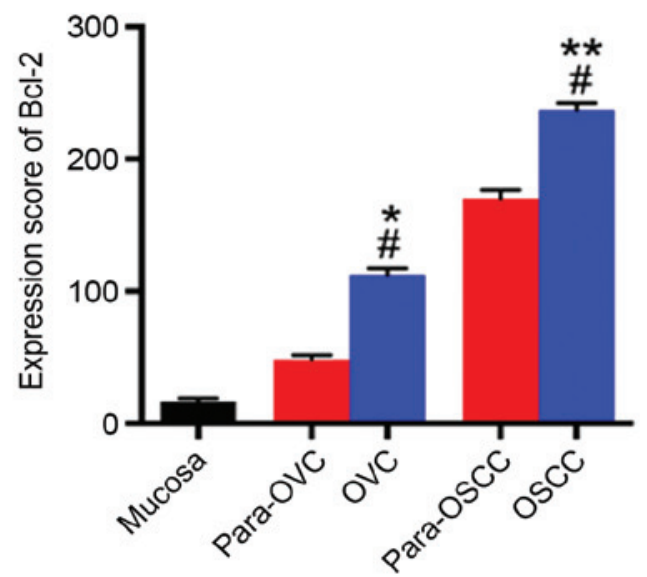

Figure 2. IHC staining of Bcl-2 in human (A) NM, (B) OVC and (C) OSCC. (D) Semi-quantitative analysis of histoscore of Bcl-2 expression in human NM, OVC and OSCC tissues. Bcl-2 expression was absent in NM tissues; however, it was expressed predominantly in the cytoplasm of OVC and OSCC cells. In $\mathrm{NM}$, OVC and OSCC, the expression of Bcl-2 intensity increased. ${ }^{\mathrm{P}}<0.05$, OVC vs. NM; ${ }^{* *} \mathrm{P}<0.01$, OSCC vs. NM; ${ }^{*} \mathrm{P}<0.05$, para-OVC vs. OVC, para-OSCC vs. OSCC, OVC vs. OSCC. Magnification, x400. NM, normal oral mucosa; OVC, oral verrucous carcinoma; para-OVC, para-tumor tissues of oral verrucous carcinoma; OSCC, oral squamous cell carcinoma; Para-OSCC, para-tumor tissues of oral squamous cell carcinoma; Bcl-2, B-cell lymphoma 2; IHC, immunohistochemistry.

cat. no. ab36707). Subsequently, a secondary biotinylated IgG antibody solution (cat. no. KIT-9710; Fuzhou Maixin Biotech Co., Ltd., Fuzhou, China) and an avidin-biotin-peroxidase reagent (OriGene Technologies, Inc.) were used to incubate the slices. The color was developed with 3,3'-diaminobenzidine and counterstained with hematoxylin. The slices were washed with phosphate-buffered saline (PBS). An incubation with PBS instead of the antibody was used for the negative control.

Staining evaluation. Each slice was scanned using an Aperio ScanScope CS scanner (Leica Microsystems, Inc., Buffalo Grove, IL, USA) with background subtraction. Aperio Quantification software version 9.1 (Leica Microsystems, Inc.) was used to quantify nuclear, membrane, or total expression levels (25). Epithelial and cancerous areas were selected for quantification. The expression scores $(3+, 2+$ and $1+$ indicated strong, medium and weak positive staining, respectively) of nuclear and membrane staining were calculated as a percentage of the positive cells using the following formula: $(3+) \times 3+(2+) \times 2+(1+) \times 1$. The expression scores of total quantification was scored as total intensity or total cell number (26). The standard controls were provided by Aperio to set the threshold for scanning of the positive cells (26-28).

Statistical analysis. Data were analyzed using the GraphPad Prism software, version 5.0 (GraphPad Software, Inc., La Jolla, CA, USA). The difference in the immunostaining between groups was determined using one-way analysis of variance followed by Tukey's or Bonferroni post-hoc tests for multiple comparisons. The correlation between the expression levels of LRIG1 and Bcl-2 was determined using two-tailed Pearson's correlation, subsequent to confirmation that the data had a Gaussian distribution. All data were expressed as the mean \pm standard error and $\mathrm{P}<0.05$ was considered to indicate a statistically significant difference.

\section{Results}

miR-181b is overexpressed in OVC tissue. The expression level of miR-181b was evaluated using qRT-PCR and was significantly greater in tissue samples from patients with $\mathrm{OVC}(\mathrm{P}<0.05)$ and OSCC $(\mathrm{P}<0.01)$ when compared with the NM tissues (Fig. 1). However, expression levels of miR-181b in OVC samples were significantly reduced when compared with OSCC samples $(\mathrm{P}<0.05)$. No significant difference was identified between the expression levels of miR-181b in the adjacent para-tumor OVC (para-OVC) and para-tumor OSCC (para-OSCC) tissue samples (Fig. 1; P>0.05); however, it was lower in comparison with to the tumor tissue samples. In addition, miR-181b expression levels were significantly greater in para-OSCC tissues when compared with NM $(\mathrm{P}<0.01)$. These findings indicated that the aberrant expression of miR-181b may be important for the initiation of OVC and its malignant progression.

Bcl-2 is upregulated in OVC tissue. miR-181b has been demonstrated to be important for the apoptosis of tumor cells 
A

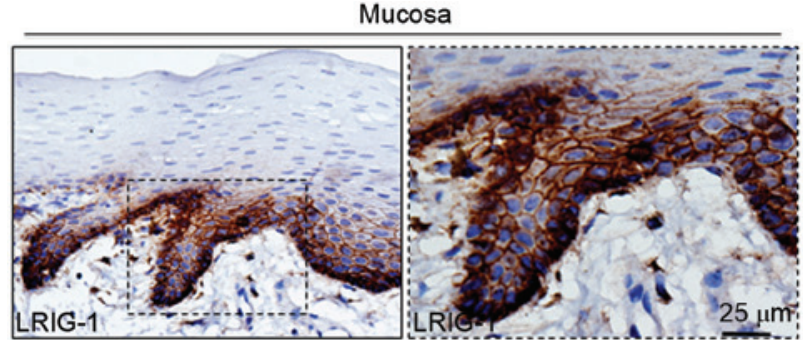

C

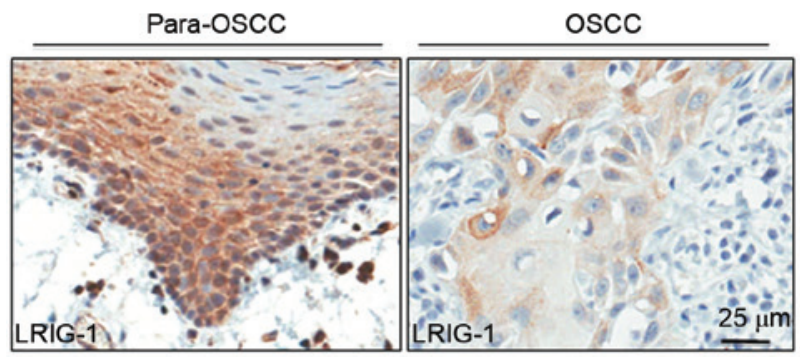

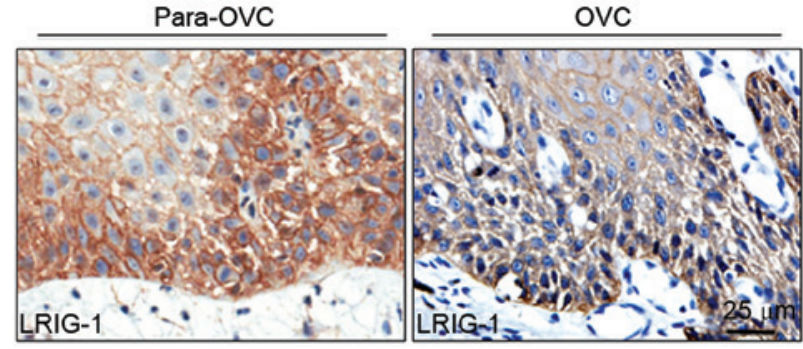

D

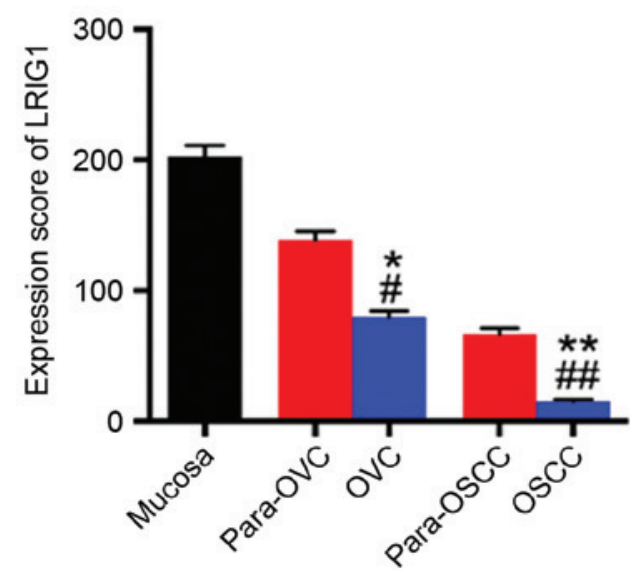

Figure 3. IHC staining of LRIG1 in human (A) NM, (B) OVC and (C) OSCC tissues. Representative IHC staining determined that LRIG1 was primarily located in the cell membrane and the cytoplasm of the tissues. Magnification, x400. (D) Semi-quantitative analysis of histoscore of LRIG1 expression in human NM, OVC and OSCC tissues. In NM, OVC and OSCC, the expression of LRIG1 intensity was decreased. ${ }^{*} \mathrm{P}<0.05, \mathrm{OVC}$ vs. NM; ${ }^{* *} \mathrm{P}<0.01, \mathrm{OSCC}$ vs. NM; ${ }^{*} \mathrm{P}<0.05$, para-OVC vs. OVC; ${ }^{\#} \mathrm{P}<0.01$, para-OSCC vs. OSCC, OVC vs. OSCC. NM, normal oral mucosa; OVC, oral verrucous carcinoma; para-OVC, para-tumor tissues of oral verrucous carcinoma; OSCC, oral squamous cell carcinoma; para-OSCC, para-tumor tissues of oral squamous cell carcinoma; LRIG1, leucine rich repeats and immunoglobulin like domains 1.

and $\mathrm{Bcl}-2$ is an established target of miR-181b (15). Therefore, $\mathrm{Bcl}-2$ expression was also subsequently examined by IHC staining (Fig. 2) in order to determine whether miR-181b targeted Bcl-2, which is an important anti-apoptotic protein (29). IHC analysis indicated that the expression level of Bcl-2 in NM tissues was significantly reduced when compared with OVC $(\mathrm{P}<0.05$; Fig. 2D) and OSCC $(\mathrm{P}<0.01$; Fig. 2D) tissues. In addition, Bcl-2 protein levels in OVC and OSCC tissues were significantly higher when compared with their corresponding adjacent para-tumor tissues (para-OVC vs. OVC, $\mathrm{P}<0.05$ and para-OSCC vs. OSCC, $\mathrm{P}<0.05$; Fig 2D). Bcl-2 expression levels in OVC tissue samples were significantly higher compared with NM tissues $(\mathrm{P}<0.05$; Fig. 2D). However, they were significantly lower when OSCC tissue samples were compared with OVC tissues $(\mathrm{P}<0.05$; Fig. 2D).

Downregulation of LRIG1 expression in OVC tissue. The expression levels of LRIG1 in OVC and OSCC were investigated by staining the mucosa, including OVC and OSCC tissue samples with an antibody targeting LRIG1 (Fig. 3). The expression was quantified by scoring the intensity of staining and the results demonstrated that the expression of LRIG1 in NM tissues was significantly greater when compared with OVC $(\mathrm{P}<0.05$; Fig. 3D) and OSCC $(\mathrm{P}<0.01$; Fig. 3D $)$ tissue samples. In addition, the expression levels of LRIG1 in para-OVC and para-OSCC samples were significantly higher compared with the tumor tissues (para-OVC vs. OVC, $\mathrm{P}<0.05$ and para-OSCC vs. OSCC, P<0.01; Fig. 3D). These results

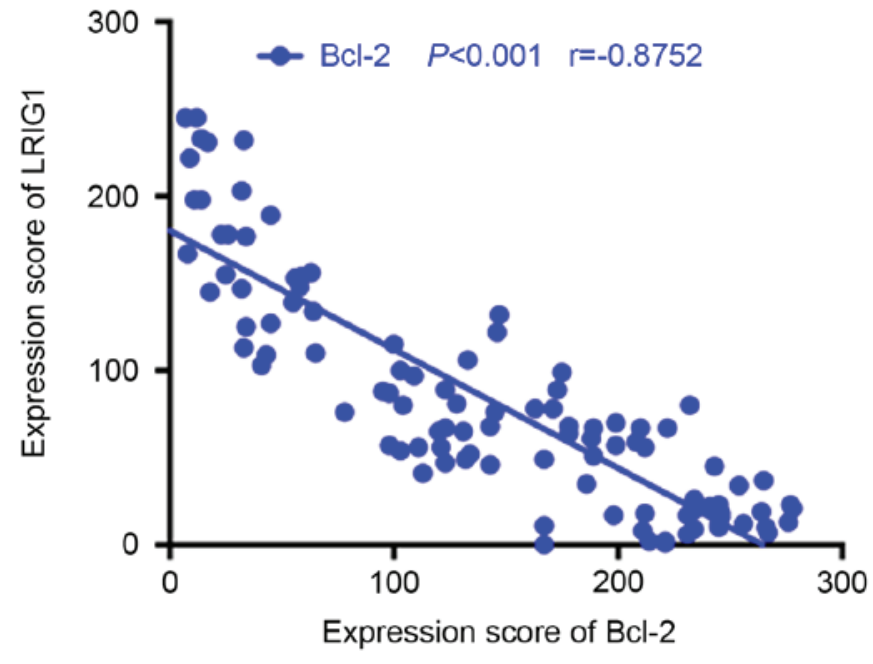

Figure 4. Correlation between LRIG1 and Bcl-2 in human NM, OVC and OSCC tissues. LRIG1 was negatively correlated with Bcl-2 expression in NM, OVC and OSCC tissues. LRIG1, leucine rich repeats and immunoglobulin like domains 1; Blc-2, B-cell lymphoma 2.

support the hypothesis that LRIG1 may act as a potential tumor suppressor gene in OVC and OSCC tissues.

Correlation of LRIGI and Bcl-2 expression in NM, OVC and OSCC tissues. The association between Bcl-2 and LRIG1 expression was determined using a two-tailed Pearson's correlation. The results indicated that LRIG1 expression was 
negatively correlated with $\mathrm{Bcl}-2$ expression in NM, OVC and OSCC tissue samples (Fig. 4; $\mathrm{r}=-0.8752 ; \mathrm{P}<0.001$ ).

\section{Discussion}

The first miRNA was characterized in Caenorhabditis elegans and reported in 1993, subsequently thousands of miRNAs have been identified (30). miRNAs regulate the expression of their target mRNAs via complementarily binding within the 3'-untranslated region of the target mRNA. This binding results in the degradation of the mRNA under conditions of perfect complementarity, or the inhibition of translation under conditions of partial complementarity (31). Abnormal miRNA expression of has been indicated in numerous types of cancer, including glioma, gastric, liver, prostate, head and neck cancer and OSCC. In terms of their function in cancer pathology miRNAs may be divided into two types, a number act as tumor promoters and others as tumor suppressors; however, both are involved in regulation of tumor cell proliferation, invasion and apoptosis (32). The miR-181s family members include miR-181a, miR-181b, miR-181c and miR-181d (33), their abnormal expression has been reported in various tumors. Upregulation of miR-181b may promote tumor cell invasion and metastasis in breast cancer (34). In hepatocellular carcinoma, the upregulation of miR-181b expression levels has been indicated to enhance the invasion, metastasis and strengthen the resistance to antitumor therapeutic agents (15).

The expression of miR-181b in OSCC tissues was higher and correlated with tumor lymph node metastasis and invasiveness in a previous study by Yang et al (20), indicating that miR-181b may have oncogenic potential (20). In the present study, miR-181b was upregulated in OVC, consistent with the previous finding in OSCC (20). However, no significant difference was identified in the expression level of miR-181b in tumor tissues compared with samples from adjacent tissues. This may be due to the exposure of the neighboring cells to same microenvironment and may indicate the importance of miR-181b in the progression of malignancy. The expression levels of miR-181b in OVC tissue were significantly lower compared with OSCC tissue. The expression levels of miR-181b in tumor-adjacent OSCC tissue samples were significantly different compared with NM tissue. These results suggest that miR-181b expression may be associated with the degree of malignancy, which is in agreement with previous studies in oral leukoplakia and dysplastic lesions $(17,18)$, as OVC is regarded as a low-grade malignant tumor, which is less prone to invasion and metastasis and has a better prognosis, in comparison with OSCC.

Bcl-2 has been identified as a target of miR-181b. In addition, it has been observed that the downregulation of miR-181b was inversely correlated with an increase in the protein levels of BCL2 family apoptosis regulator (MCL1) and Bcl-2, which are target genes in chronic lymphocytic leukemia (35). In astrocytes, miR-181 affects the cellular apoptosis and mitochondrial function by targeting multiple Bcl-2 family members, including BCL2 like 11, MCL1 and Bcl-2 (36,37). It has been reported that miR-181b acts as a tumor suppressor gene in gliomas and that its downregulation may lead to tumor growth, inhibit tumor cell invasion and promote apoptosis (38). However, a previous study demonstrated that the overexpression of miR-181a resulted in significant downregulation of the Bcl-2 protein levels in malignant glioma cells exposed to radiation treatment; thus, miR-181 may be a potential target for enhancing the effect of radiotherapy by regulating Bcl-2 expression (19). In addition, the overexpression of miR-181b in gastric cancer may enhance migration and invasion of gastric cancer cells, thus promoting the occurrence and development of gastric cancer (16). Through employing the miRanda online database (www.microrna.org/microrna/getMirnaForm.do), LRIG1 was identified as a potential target of miR-181b. A previous study has determined that LRIG1 is a tumor suppressor gene and its expression is correlated with the grade of the malignancy (23). The present study highlighted consistent trends in expression of miR-181b and Bcl-2 in OVC and OSCC tissues, where their expression levels were significantly higher compared with NM tissue. This is consistent with previous studies by Singh et al (39) and Su et al (40) in OSCC tissues. However, this is not consistent with the results of previous study in chronic lymphoid leukemia (35). In addition, the present study determined that LRIG1 expression is important in OVC and OSCC tissues. Bioinformatics prediction has indicated LRIG1 is one of the target genes of miR-181b, in addition to Bcl-2.

LRIG1 expression has also been associated with Bcl-2 expression in human ependymomas (41). This may suggest the existence of an alternative regulation pathway. Therefore, it is possible that miR-181b may indirectly regulate Bcl-2 expression via a signaling pathway that involves LRIG1. The current study determined that there were decreased expression levels of LRIG1 in OVC tissues. In addition, the expression levels of Bcl-2 were negatively correlated with LRIG1 expression levels in NM, OVC and OSCC tissues. LRIG1 may be have anti-apoptotic functions in OVC. Initially, it was determined that miR-181b had increased expression levels in OVC. The specific molecular mechanism that is responsible for the changes in miR-181b and LRIG1 expression levels requires further experimental verification.

In conclusion, the expression of miR-181b and $\mathrm{Bcl}-2$ in OVC tissues was significantly higher compared with NM tissue; however lower when OVC was compared with OSCC tissues. The expression levels of the target of miR-181b, LRIG1, in OVC tissue were significantly reduced when compared with NM. However, LRIG1 expression levels in were determined to be higher in OVC tissues compared with OSCC tissues. The expression levels of $\mathrm{Bcl}-2$ were negatively correlated with the expression of LRIG1 in NM, OVC and OSCC tissues. Therefore, LRIG1 may have an anti-apoptotic function in OVC.

\section{Acknowledgements}

The present study was supported by the Fundamental Research Funds for the Central Universities of Central South University (grant nos. 2012zzts135 and 2016zzts117), The Nature Sciences Foundation of Qinghai Province (grant no. 2013-z-908) and The Nature Sciences Foundation of Hunan Province (grant no. S2013J504B).

\section{References}

1. Ackerman LV: Verrucous carcinoma of the oral cavity. Surgery 23: 670-678, 1948. 
2. Arduino PG, Carrozzo M, Pagano M, Gandolfo S and Broccoletti R: Verrucous oral carcinoma: Clinical findings and treatment outcomes in 74 patients in Northwest Italy. Minerva Stomatol 57: 335-339, 339-341, 2008.

3. Impola U, Uitto VJ, Hietanen J, Hakkinen L, Zhang L, Larjava H, Isaka K and Saarialho-Kere U: Differential expression of matrilysin-1 (MMP-7), 92 kD gelatinase (MMP-9) and metalloelastase (MMP-12) in oral verrucous and squamous cell cancer. J Pathol 202: 14-22, 2004.

4. Medina JE, Dichtel W and Luna MA: Verrucous-squamous carcinomas of the oral cavity. A clinicopathologic study of 104 cases. Arch Otolaryngol 110: 437-440, 1984.

5. Walvekar RR, Chaukar DA, Deshpande MS, Pai PS, Chaturvedi P, Kakade A, Kane SV and D'Cruz AK: Verrucous carcinoma of the oral cavity: A clinical and pathological study of 101 cases. Oral Oncol 45: 47-51, 2009.

6. Yeh CJ: Treatment of verrucous hyperplasia and verrucous carcinoma by shave excision and simple cryosurgery. Int J Oral Maxillofac Surg 32: 280-283, 2003.

7. Patel KR, Chernock RD, Zhang TR, Wang X, El-Mofty SK and Lewis JS Jr: Verrucous carcinomas of the head and neck, including those with associated squamous cell carcinoma, lack transcriptionally active high-risk human papillomavirus. Hum Pathol 44: 2385-2392, 2013.

8. Quan H, Tang Z, Zhao L, Wang Y, Liu O, Yao Z and Zuo J: Expression of $\alpha \mathrm{B}$-crystallin and its potential anti-apoptotic role in oral verrucous carcinoma. Oncol Lett 3: 330-334, 2012

9. Lin HP, Wang YP and Chiang CP: Expression of p53, MDM2, p21, heat shock protein 70, and HPV 16/18 E6 proteins in oral verrucous carcinoma and oral verrucous hyperplasia. Head Neck 33: 334-340, 2011.

10. Wang YH, Tian X, Liu OS, Fang XD, Quan HZ, Xie S, Gao S and Tang ZG: Gene profiling analysis for patients with oral verrucous carcinoma and oral squamous cell carcinoma. Int J Clin Exp Med 7: 1845-1852, 2014.

11. Liu O, Zhang H, Wang Y, Quan H, Zhang J, Zhou J, Zuo J, Tang J, Fang X, Wang W, et al: Stereology study of oral verrucous carcinoma. J Buon 17: 343-349, 2012.

12. Gomes CC and Gomez RS: MicroRNA and oral cancer: Future perspectives. Oral Oncol 44: 910-914, 2008.

13. Allegra A, Alonci A, Campo S, Penna G, Petrungaro A, Gerace D and Musolino C: Circulating microRNAs: New biomarkers in diagnosis, prognosis and treatment of cancer (review). Int J Oncol 41: 1897-1912, 2012.

14. Etheridge A, Lee I, Hood L, Galas D and Wang K: Extracellular microRNA: A new source of biomarkers. Mutat Res 717: 85-90, 2011

15. Wang B, Hsu SH, Majumder S, Kutay H, Huang W, Jacob ST and Ghoshal K: TGFbeta-mediated upregulation of hepatic miR-181b promotes hepatocarcinogenesis by targeting TIMP3. Oncogene 29: 1787-1797, 2010

16. Guo JX, Tao QS, Lou PR, Chen XC, Chen J and Yuan GB miR-181b as a potential molecular target for anticancer therapy of gastric neoplasms. Asian Pac J Cancer Prev 13: 2263-2267, 2012.

17. Cervigne NK, Reis PP, Machado J, Sadikovic B, Bradley G, Galloni NN, Pintilie M, Jurisica I, Perez-Ordonez B, Gilbert R, et al: Identification of a microRNA signature associated with progression of leukoplakia to oral carcinoma. Hum Mol Genet 18: 4818-4829, 2009.

18. Brito JA, Gomes CC, Guimarães AL, Campos K and Gomez RS Relationship between microRNA expression levels and histopathological features of dysplasia in oral leukoplakia. J Oral Pathol Med 43: 211-216, 2014.

19. Chen G, Zhu W, Shi D, Lv L, Zhang C, Liu P and $\mathrm{Hu}$ W: MicroRNA-181a sensitizes human malignan glioma U87MG cells to radiation by targeting Bcl-2. Oncol Rep 23: 997-1003, 2010.

20. Yang CC, Hung PS, Wang PW, Liu CJ, Chu TH, Cheng HW and Lin SC: miR-181 as a putative biomarker for lymph-node metastasis of oral squamous cell carcinoma. J Oral Pathol Med 40: 397-404, 2011

21. Barinaga M: Death by dozens of cuts. Science 280: 32-34, 1998.
22. Ding J, Liu B, He Y, Yuan X, Tian D, Ji B, Wang L, Wu L, Dong $\mathrm{H}$, Wang $\mathrm{J}$, et al: LRIG1 improves chemosensitivity through inhibition of BCL-2 and MnSOD in glioblastoma. Cell Biochem Biophys 71: 27-33, 2015.

23. Xu Y, Soo P, Walker F, Zhang HH, Redpath N, Tan CW, Nicola NA, Adams TE, Garrett TP, Zhang JG and Burgess AW: LRIG1 extracellular domain: Structure and function analysis. J Mol Biol 427: 1934-1948, 2015.

24. Livak KJ and Schmittgen TD: Analysis of relative gene expression data using real-time quantitative PCR and the 2(-Delta Delta C(T)) method. Methods 25: 402-408, 2001.

25. Huang CF, Zhang L, Ma SR, Zhao ZL, Wang WM, He KF, Zhao YF, Zhang WF, Liu B and Sun ZJ: Clinical significance of Keap1 and Nrf2 in oral squamous cell carcinoma. PLoS One 8: e83479, 2013

26. Sun ZJ, Zhang L, Hall B, Bian Y, Gutkind JS and Kulkarni AB: Chemopreventive and chemotherapeutic actions of mTOR inhibitor in genetically defined head and neck squamous cell carcinoma mouse model. Clin Cancer Res 18: 5304-5313, 2012.

27. Bian Y, Hall B, Sun ZJ, Molinolo A, Chen W, Gutkind JS Waes CV and Kulkarni AB: Loss of TGF- $\beta$ signaling and PTEN promotes head and neck squamous cell carcinoma through cellular senescence evasion and cancer-related inflammation. Oncogene 31: 3322-3332, 2012

28. Ma SR, Wang WM, Huang CF, Zhang WF and Sun ZJ: Anterior gradient protein 2 expression in high grade head and neck squamous cell carcinoma correlated with cancer stem cell and epithelial mesenchymal transition. Oncotarget 6: 8807-8821,2015.

29. Zhu W, Shan X, Wang T, Shu Y and Liu P: miR-181b modulates multidrug resistance by targeting BCL 2 in human cancer cell lines. Int J Cancer 127: 2520-2529, 2010.

30. Wu BH, Xiong XP, Jia J and Zhang WF: MicroRNAs: New actors in the oral cancer scene. Oral Oncol 47: 314-319, 2011.

31. Esquela-Kerscher A and Slack FJ: Oncomirs-microRNAs with a role in cancer. Nat Rev Cancer 6: 259-269, 2006.

32. Lynam-Lennon N, Maher SG and Reynolds JV: The roles of microRNA in cancer and apoptosis. Biol Rev Camb Philos Soc 84: 55-71, 2009

33. Henao-Mejia J, Williams A, Goff LA, Staron M, Licona-Limón P, Kaech SM, Nakayama M, Rinn JL and Flavell RA: The microRNA miR-181 is a critical cellular metabolic rheostat essential for NKT cell ontogenesis and lymphocyte development and homeostasis. Immunity 38: 984-997, 2013.

34. Neel JC and Lebrun JJ: Activin and TGF $\beta$ regulate expression of the microRNA-181 family to promote cell migration and invasion in breast cancer cells. Cell Signal 25: 1556-1566, 2013.

35. Visone R, Veronese A, Rassenti LZ, Balatti V, Pearl DK, Acunzo M, Volinia S, Taccioli C, Kipps TJ and Croce CM: miR-181b is a biomarker of disease progression in chronic lymphocytic leukemia. Blood 118: 3072-3079, 2011.

36. Ouyang YB, Lu Y, Yue S and Giffard RG: miR-181 targets multiple $\mathrm{Bcl}-2$ family members and influences apoptosis and mitochondrial function in astrocytes. Mitochondrion 12: 213-219, 2012.

37. Lu F, Zhang J, Ji M, Li P, Du Y, Wang H, Zang S, Ma D, Sun X and Ji C: miR-181b increases drug sensitivity in acute myeloid leukemia via targeting HMGB1 and Mcl-1. Int J Oncol 45: 383-392, 2014.

38. Shi L, Cheng Z, Zhang J, Li R, Zhao P, Fu Z and You Y: hsa-mir-181a and hsa-mir-181b function as tumor suppressors in human glioma cells. Brain Res 1236: 185-193, 2008.

39. Singh BB, Chandler FW Jr, Whitaker SB and Forbes-Nelson AE: Immunohistochemical evaluation of bcl-2 oncoprotein in oral dysplasia and carcinoma. Oral Surg Oral Med Oral Pathol Oral Radiol Endod 85: 692-698, 1998.

40. Su L, Wang Y, Xiao M, Lin Y and Yu L: Up-regulation of survivin in oral squamous cell carcinoma correlates with poor prognosis and chemoresistance. Oral Surg Oral Med Oral Pathol Oral Radiol Endod 110: 484-491, 2010.

41. Yi W, Haapasalo H, Holmlund C, Järvelä S, Raheem O, Bergenheim AT, Hedman H and Henriksson R: Expression of leucine-rich repeats and immunoglobulin-like domains (LRIG) proteins in human ependymoma relates to tumor location, WHO grade and patient age. Clin Neuropathol 28: 21-27, 2009. 